\title{
Parental Mental Health in Childhood as a Risk Factor for Anxiety and Depression among People Seeking Help for Tinnitus and Hyperacusis
}

DOI: $10.3766 /$ jaaa. 18001

\author{
Hashir Aazh* \\ Ali A. Danesh $\dagger$ \\ Brian C. J. Moore
}

\begin{abstract}
Background: Parental mental illness is a risk factor for mental health disorders in the offspring. However, the relationship between parental illness in childhood and mental health disorders in adulthood among patients with tinnitus and/or hyperacusis is not known.
\end{abstract}

Purpose: The aim was to explore the relationship between parental mental health in childhood and anxiety and depression for patients experiencing tinnitus and/or hyperacusis.

Research Design: This was a retrospective cross-sectional study with a correlational design.

Study Sample: Two hundred eighty-seven consecutive patients who attended a Tinnitus and Hyperacusis Therapy Specialist Clinic in the United Kingdom were included. Their average age was 52.5 years.

Data Collection and Analysis: The association was explored between anxiety and depression measured via the Generalized Anxiety Disorder questionnaire (GAD-7) and the Patient Health questionnaire (PHQ-9) and responses to the question "While you were growing up during the first 18 years of life did your parent(s) have depression or mental illness?"

Results: Thirty-nine percent of patients (111/287) responded "yes" to the question about their parents' mental health, which is about double the incidence in the general population. Regression analysis showed that parental mental illness significantly increased the risk of anxiety and depression, with unadjusted odds ratios (ORs) of 2.7 (95\% confidence interval [Cl]: 1.5-4.9, $p=0.001)$ for the PHQ-9 and 2.6 (95\% Cl: $1.4-4.8, p=0.002)$ for the GAD-7. However, when the models were adjusted for the effects of age, gender, tinnitus handicap as measured via the Tinnitus Handicap Inventory, hyperacusis handicap as measured via the Hyperacusis questionnaire, uncomfortable loudness levels, GAD-7 scores (for the depression model only), and PHQ-9 scores (for the anxiety model only), parental mental health was only significantly associated with depression, with an OR of $2.7(95 \% \mathrm{Cl}$ : $1.08-6.7, p=0.033)$.

Conclusions: Audiologists offering tinnitus and hyperacusis rehabilitation should screen patients for parental mental illness in childhood, especially for those with comorbid depression, and make onward referral to appropriate mental health services when needed. Future research should analyze the breadth and type of adverse childhood experiences among patients with tinnitus and hyperacusis and their relationship with mental problems and treatment efficacy.

Key Words: adverse childhood experiences, hyperacusis, parental mental health, parental separation, tinnitus

Abbreviations: $\mathrm{ACE}=$ adverse childhood experiences; BSA = British Society of Audiology; CBT = cognitive behavioral therapy; $\mathrm{Cl}=$ confidence interval; $\mathrm{GAD}=$ Generalized Anxiety Disorder questionnaire; $\mathrm{HQ}=$ Hyperacusis questionnaire; $\mathrm{OR}=$ odds ratio; $\mathrm{PHQ}=$ Patient Health questionnaire; PTA = pure-tone average audiometric threshold; SD = standard deviation; THI = Tinnitus Handicap

*Audiology Department, Royal Surrey County Hospital NHS Foundation Trust, Egerton Road, Guildford, United Kingdom; $†$ Department of Communication Sciences and Disorders, Florida Atlantic University, Boca Raton, FL; †Department of Experimental Psychology, University of Cambridge, Cambridge, United Kingdom

Corresponding author: Hashir Aazh, Audiology Department, Royal Surrey County Hospital NHS Foundation Trust, Guildford GU2 7XX, United Kingdom; Email: hashir.aazh@nhs.net 
Inventory; THTSC = Tinnitus and Hyperacusis Therapy Specialist Clinic; ULL = uncomfortable loudness level; ULLmin = across-frequency average ULL for the ear with the lower ULL

\section{INTRODUCTION}

$\mathrm{T}$ innitus is the sensation of sound without any external acoustic source. Hyperacusis is intolerance of everyday sounds that cause significant distress and impairment in social, occupational, recreational, and other day-to-day activities (Aazh et al, 2016). There are several reports suggesting a high prevalence of psychological problems among patients suffering from tinnitus and hyperacusis (Andersson et al, 2004; Juris et al, 2013; Pinto et al, 2014; Schecklmann et al, 2014; Pattyn et al, 2016; Paulin et al, 2016; Aazh and Moore, 2017a,d; 2018b,d). A recent study conducted under the UK National Health Service reported that $33 \%(164 / 499)$ of such patients had borderline abnormal or abnormal scores on the depression subscale of the Hospital Anxiety and Depression Scale (Zigmond and Snaith, 1983). For the anxiety subscale of the Hospital Anxiety and Depression Scale, $57 \%$ of patients (284/499) had borderline abnormal or abnormal scores (Aazh and Moore, 2017a). At present, there is no definitive cure for tinnitus and hyperacusis. However, audiologists apply a wide range of rehabilitative approaches (e.g., audiologist-delivered cognitive behavioral therapy (CBT), sound therapy, provision of hearing aids, education, and counseling) to help patients minimize the emotional problems caused by their tinnitus and/or hyperacusis (Tyler et al, 1989; Jastreboff and Jastreboff, 2003; Aazh et al, 2008; Aazh et al, 2014; Henry et al, 2015; Aazh and Allott, 2016; Aazh et al, 2016; Aazh and Moore, 2016; 2018c). Therefore, it is important to explore factors contributing to the emotional problems commonly experienced by patients who suffer from tinnitus and/or hyperacusis.

There is a large body of epidemiological evidence showing that adverse childhood experiences (ACE) play a major lifelong role in mental and physical health problems (Dube et al, 2001; Dong et al, 2004; Anda et al, $2006 ; 2007 ; 2008 ; 2009 ; 2010)$. The relationship between $\mathrm{ACE}$ and mental health in adulthood varies across populations (Alcala et al, 2017; Lee and Chen, 2017; Jones et al, 2018). For example, Bohman et al (2017) reported that among people with depression, ACE was significantly associated with bipolar disorder (regression coefficient, $b=0.86, p=0.048$ ), whereas there was no significant relationship between ACE and bipolar disorder for a nondepressed control group $(b=0.45$, $p=0.72$ ). It is not known whether patients who develop tinnitus and/or hyperacusis in adulthood are more likely to exhibit anxiety and depression if they have a history of ACE than if they have no such history.
ACE can include exposure to different forms of abuse (physical, emotional, or sexual) and various aspects of household dysfunction (substance abuse in the family, parental mental illness, mother treated violently, imprisoned household member, or parental separation) (Felitti et al, 1998). Parental separation and parental mental illness are important forms of ACE, with prevalences of $23 \%$ and $19 \%$, respectively (Anda et al, 2006). Aazh et al (2018) compared tinnitus and hyperacusis handicap as measured via the Tinnitus Handicap Inventory (THI) (Newman et al, 1998) and the Hyperacusis questionnaire (HQ) (Khalfa et al, 2002) for patients with and without a history of parental separation and parental mental illness in childhood $(\mathrm{n}=184)$. There were no associations between parental separation and scores for the THI and HQ. However, scores for the THI and HQ were worse for the group that reported mental illness in their parents than for the group that did not $(p<0.01)$. Further analysis of the data suggested that parental mental illness was not significantly related to THI scores. However, it was significantly related to the risk of hyperacusis (odds ratio [OR] after adjusting for age and gender: 2.05, $p=$ 0.026). The adjusted OR for a subgroup of patients with a diagnosis of hyperacusis was 6.7 ( $p=0.011$ ), indicating a stronger relationship for this subgroup.

Aazh et al (2018) did not assess whether patients who develop tinnitus and/or hyperacusis later on in their life had a greater risk of developing anxiety and depression if their parents had poor mental health than if they did not. Although tinnitus and hyperacusis are often associated with symptoms of anxiety and depression (Langguth et al, 2011), not everyone with abnormal scores on the THI and/or HQ presents with symptoms of anxiety and depression (Aazh and Moore, 2017d).

Exploring the possible link between poor parental mental health in childhood and anxiety and depression in adulthood is important because, if a significant relationship exists, the presence of anxiety and depression in patients with tinnitus and/or hyperacusis could be an indicator of ACE. Research studies suggest that, in general, patients with a history of ACE need more complex and in-depth psychological treatments for their mental health should they develop emotional problems (Pigeon et al, 2009; Kajeepeta et al, 2015). There is a need for identification of symptoms that would suggest a history of ACE so that proper treatment and care can be provided (Kajeepeta et al, 2015).

At the Tinnitus and Hyperacusis Therapy Specialist Clinic (THTSC), patients are routinely asked about their childhood experiences in addition to completing 
a wide range of self-report questionnaires assessing tinnitus, hyperacusis, anxiety, and depression. This provided a unique opportunity to explore whether parental mental illness in childhood plays a role in the development of anxiety and depression among patients who seek help for their tinnitus and/or hyperacusis. To the authors' knowledge, there is no study in the literature assessing this question.

In summary, the aim of this study was to explore the relationship between parental mental health in childhood and anxiety and depression symptoms for adult patients with tinnitus and hyperacusis, as seen in an National Health Service specialist clinic.

\section{METHOD}

\section{Study Design and Patients}

This was a retrospective cross-sectional study with a correlational design conducted at the THTSC, Royal Surrey County Hospital, Guildford, UK. The data were based on 287 consecutive patients who attended the THTSC between April 2017 and March 2018 and for whom audiological measures and selfreport questionnaires had been obtained. The average age of the patients was 52.5 years (standard deviation $[\mathrm{SD}]=15.5$ years, range from 18 to 87 years). Forty-nine percent (141/287) of the patients were male.

Demographic data for the patients and the outcomes of their audiological investigations and their self-report questionnaires were extracted from records held at the THTSC.

\section{Parental Mental Health}

Parental mental health was assessed using a question adopted from the questionnaire for ACE (Felitti et al, 1998; Anda et al, 2006). The question was "While you were growing up during the first 18 years of life, did your parent(s) have depression or mental illness?" (p. 247; Felitti et al, 1998). The response alternatives were "yes" or "no." The test-retest reliability for this question is moderate (Cohen's kappa $=0.48$, standard error $=0.052,95 \%$ confidence interval $[\mathrm{CI}]=0.37-0.58$ ) (Dube et al, 2004).

\section{Tinnitus and Hyperacusis Handicap}

Tinnitus was assessed using the THI (Newman et al, 1996). This has 25 items, and response choices are "no" (0 points), "sometimes" ( 2 points), and "yes" (4 points). The overall score ranges from 0 to 100 . Scores from 0 to 16 indicate no handicap, from 18 to 36 mild handicap, from 38 to 56 moderate handicap, and from 58 to 100 severe handicap (Newman et al, 1996).
Hyperacusis was assessed using the HQ (Khalfa et al, 2002). This has 14 items and the response choices are "no" (0 points); "yes, a little" (1 points); "yes, quite a lot" ( 2 points); and "yes, a lot" (3 points). The overall score ranges from 0 to 42 . Scores of 22 or more were taken as indicating the presence of hyperacusis (Aazh and Moore, 2017b).

\section{Depression and Anxiety}

Depression was assessed using the Patient Health questionnaire (PHQ-9; Kroenke et al, 2001), which has nine items. Response options are as follows: not at all (0), several days (1), more than half the days (2), and nearly every day (3). The total score ranges from 0 to 27. The recommended cutoff for depression in the UK mental health system is a score of 10 or above (IAPT, 2011).

Anxiety was assessed using the Generalized Anxiety Disorder questionnaire (GAD-7; Spitzer et al, 2006), which has seven items. Patients were asked how often during the last two weeks they had been bothered by each symptom. Response options are as follows: not at all (0), several days (1), more than half the days (2), and nearly every day (3). The total score ranges from 0 to 21 . The recommended cutoff score for generalized anxiety in the UK mental health system is a score of 8 or above (IAPT, 2011).

\section{Audiological Investigations}

Audiological measures were as follows:

- Pure-tone audiogram measured using the procedure recommended by the British Society of Audiology (BSA, 2011a), but with some modifications proposed by Aazh and Moore (2017c) to avoid any discomfort. The starting presentation level at $0.25,0.5,2,3,4,6$, and $8 \mathrm{kHz}$ was equal to the measured audiometric threshold at the adjacent frequency (e.g., if the threshold at $1 \mathrm{kHz}$ was $20 \mathrm{~dB} \mathrm{HL}$, the starting level for measuring the threshold at $2 \mathrm{kHz}$ was $20 \mathrm{~dB} \mathrm{HL}$, instead of $50 \mathrm{~dB}$ HL as recommended by the BSA). The severity of hearing loss was categorized based on the values of the pure-tone average (PTA) across the frequencies $0.25,0.5,1,2$, and $4 \mathrm{kHz}$, as recommended by the BSA (2011a): mild (20-40 dB HL), moderate (41-70 dB HL), severe (71-95 dB HL), and profound (over $95 \mathrm{~dB} \mathrm{HL}$ ).

- Uncomfortable Loudness Levels (ULLs) measured following the BSA recommended procedure (BSA, 2011b), but with the modifications proposed by Aazh and Moore (2017c), to avoid any discomfort. The starting presentation level was equal to the measured audiometric threshold at the test frequency. In addition, levels $>80 \mathrm{~dB} \mathrm{HL}$ were not used. If the 
ULL was not reached at $80 \mathrm{~dB}$ HL, the ULL at the test frequency was recorded as $85 \mathrm{~dB}$ HL. When the average ULL at $0.25,0.5,1,2,4$, and $8 \mathrm{kHz}$ for the ear with the lower average ULL, which is called ULLmin, was $\leq 77 \mathrm{~dB}$ HL, hyperacusis was deemed to be present (Aazh and Moore, 2017b).

\section{Ethical Approval}

This study was part of a larger research project approved by the South West-Cornwall and Plymouth Research Ethics Committee and the Research and Development department at the Royal Surrey County Hospital.

\section{Data Analysis}

The data were anonymized before statistical analysis. Descriptive statistics (means and SDs) for the characteristics of the patients and scores for the self-report questionnaires were calculated. The Wilcoxon ranksum (Mann-Whitney) test was used to compare the scores for the PHQ-9 and GAD-7 between patients grouped according to whether or not their parents had mental illness. Statistically significant differences across groups were explored using logistic regression to assess whether parental mental health (independent variable) predicted abnormal scores on the PHQ-9 and GAD-7 (dependent variables). ORs and their 95\% CI were calculated. The ORs were calculated both unadjusted and adjusted for age, gender, tinnitus handicap as measured via the THI, hyperacusis as measured via ULLmin and $\mathrm{HQ}$ scores, GAD-7 scores (for the depression model only), and PHQ-9 scores (for the anxiety model only).

Some of the patients did not complete all of the selfreport questionnaires or audiological investigations. Hence, the analyses were restricted to patients for whom there were complete data on all variables required for a particular analysis. The number of patients included in each analysis (n) is reported. The $p$-value required for statistical significance was set at $p<$ 0.05. The STATA program (version 13; StataCorp LP, College Station, TX) was used for statistical analyses.

\section{RESULTS}

\section{Characteristics of the Study Population}

Table 1 shows means and SDs of scores on the THI, HQ, PHQ-9 and GAD-7, the mean PTA for the better ear, and the mean value of ULLmin.

Based on the PTA for the better ear, $60 \%$ of patients (155 of 258 with audiograms) had no hearing loss, $34 \%$ (87/258) had mild hearing loss, 6\% (15/258) had moderate hearing loss, and $0.4 \%$ (1/258) had profound hearing loss. Based on the values of ULLmin, $41 \%$ of patients (81/196) had ULLs of $77 \mathrm{~dB}$ HL or below, which indicates hyperacusis.

Based on HQ scores, 36\% (101/283) of patients experienced hyperacusis. Based on THI scores, $7.5 \%$ of patients (21/279) had no tinnitus handicap, 25\% (70/279) had mild tinnitus handicap, 27\% (76/279) had moderate tinnitus handicap, and 40\% (112/279) had severe tinnitus handicap. Based on scores for the PHQ-9, 42\% (119/ 283) of patients experienced depression symptoms. Based on scores for the GAD-7, 47\% (133/281) of patients experienced anxiety symptoms.

\section{Parental Mental Health and its Relationship with Anxiety and Depression}

Thirty-nine percent of patients (111/287) reported that during their first 18 years of life, their parent(s) suffered from a mental illness. The mean PHQ-9 score was 11.4 $(\mathrm{SD}=6.7)$ for patients whose parents had mental illness and $7.6(\mathrm{SD}=6.4)$ for patients whose parents did not have mental illness. This difference was statistically significant $(p<0.001)$. The mean GAD-7 score was $10(\mathrm{SD}=5.9)$ for patients whose parents had mental illness and 7.2 $(\mathrm{SD}=6.2)$ for patients whose parents did not have mental illness. This difference was statistically significant ( $p=0.001$ ).

Tables 2 and 3 show the results of the logistic regression analyses of factors predicting abnormal scores on the PHQ-9 and GAD-7, respectively. Only patients who had complete data for all variables in the model were included in the regression analyses $(\mathrm{n}=189)$. Columns 2 and 3 show the unadjusted ORs and $p$ values

Table 1. Means and SDs of the PTA Across $0.25,0.5,1,2$, and $4 \mathrm{kHz}$ for the Better Ear, Average ULL at 0.25, 0.5, 1, 2, 4, and $8 \mathrm{kHz}$ for the Ear with the ULLmin, and Scores on THI, HQ, PHQ-9, and GAD-7

\begin{tabular}{llll}
\hline Questionnaire & $n$ & Mean & SD \\
\hline PTA for the better ear (dB HL) & 258 & 19 & 74 \\
ULLmin (dB HL) & 196 & 50 & 13 \\
THI & 279 & 18 & 24 \\
HQ & 283 & 9.0 & 10 \\
PHQ-9 & 283 & 8.3 & 6.8 \\
GAD-7 & 281 & 6.3 \\
\hline
\end{tabular}

Note: The number of patients for whom data were available for each measure is indicated by $\mathrm{n}$. 
Table 2. Logistic Regression Model Showing the OR for the Presence or Absence of Depression as Measured via the PHQ-9 (Dependent Variable)

\begin{tabular}{|c|c|c|c|c|}
\hline & Unadjusted OR (95\% Cl) & $p$ & Adjusted OR (95\% Cl) & $p$ \\
\hline \multicolumn{5}{|l|}{ Parental mental illness } \\
\hline No & 1.0 & & 1.0 & \\
\hline Yes & $2.7(1.5-4.9)$ & 0.001 & $2.7(1.08-6.7)$ & 0.033 \\
\hline \multicolumn{5}{|l|}{ Tinnitus handicap category } \\
\hline No handicap (THI score 0-16) & 1.0 & & 1.0 & \\
\hline Mild (THI score 18-36) & $1.09(0.1-11.4)$ & 0.94 & $0.98(0.08-12.2)$ & 0.98 \\
\hline Moderate (THI score 38-56) & $8.6(1.05-70.6)$ & 0.044 & $4.6(0.5-45.3)$ & 0.19 \\
\hline Severe (THI score 58-100) & $52.9(6.5-430)$ & $<0.001$ & $20.1(2-199.4)$ & 0.011 \\
\hline \multicolumn{5}{|l|}{ Hyperacusis handicap } \\
\hline No $(H Q<22)$ & 1.0 & & 1.0 & \\
\hline Yes $(H Q \geq 22)$ & $4.7(2.5-8.8)$ & $<0.001$ & $2.5(0.97-6.4)$ & 0.057 \\
\hline \multicolumn{5}{|l|}{ Hyperacusis } \\
\hline No (ULLmin >77 dB HL) & 1.0 & & 1.0 & \\
\hline Yes (ULLmin $\leq 77 \mathrm{~dB} \mathrm{HL})$ & $2.3(1.25-4.11)$ & 0.007 & $1.1(0.41-3.1)$ & 0.83 \\
\hline \multicolumn{5}{|l|}{ Anxiety } \\
\hline Normal (GAD-7 < 8) & 1.0 & & 1.0 & \\
\hline Abnormal (GAD-7 $\geq 8$ ) & $21.2(9.98-44.99)$ & $<0.001$ & $6.8(2.8-16.2)$ & $<0.001$ \\
\hline
\end{tabular}

Note: Unadjusted OR values and adjusted OR values and their $95 \% \mathrm{Cl}$ are presented $(\mathrm{n}=189)$. The adjusted OR takes into account the effects of age and gender in addition to the effects of other variables in the model. Variables included in the model were presence or absence of parental mental illness and categories based on scores for the THI and HQ, ULLmin (average ULL across 0.25, 0.5, 1, 2, 4, and $8 \mathrm{kHz}$ for the ear with the lower average ULL) and scores for the GAD-7. Significant $p$ values are indicated in bold font.

and columns 4 and 5 show ORs adjusted for the effects of other variables in the model and the effects of age and gender.

Table 2 (columns 2 and 3) shows that the risk of depression, indicated by a PHQ-9 score $\geq 10$, increased 2.7-fold for patients with a childhood history of parental mental illness $(p=0.001)$. When the model was adjusted for the effects of other variables in the model plus age and gender (columns 4 and 5, Table 2), the relationship between parental mental illness and depression was almost unchanged and was statistically significant. The adjusted OR was 2.7 (95\% CI: $1.08-6.7, p=0.033$ ). In the adjusted model, in addition to parental mental illness, the risk of depression was increased by a factor of 20.1 ( $p=0.011$ ) for those with severe tinnitus handicap (THI score 58-100) and by a factor of $6.8(p<0.001)$ for those with anxiety (GAD-7 $\geq 8$ ). The likelihood of hyperacusis as diagnosed by ULLmin $\leq 77 \mathrm{~dB} \mathrm{HL}$ was significantly associated with an abnormal score on the PHQ-9 (unadjusted OR $=2.3, p=0.007$ ). However,

Table 3. As Table 2, but Showing the OR for the Presence or Absence of Anxiety as Measured via the GAD-7

\begin{tabular}{|c|c|c|c|c|}
\hline & Unadjusted OR $(95 \% \mathrm{Cl})$ & $p$ & Adjusted OR (95\% Cl) & $p$ \\
\hline \multicolumn{5}{|l|}{ Parental mental illness } \\
\hline No & 1.0 & & 1.0 & \\
\hline Yes & $2.6(1.4-4.8)$ & 0.002 & $2.1(0.89-4.9)$ & 0.088 \\
\hline \multicolumn{5}{|l|}{ Tinnitus handicap category } \\
\hline No handicap (THI score 0-16) & 1.0 & & 1.0 & \\
\hline Mild (THI score 18-36) & $0.89(0.16-5.2)$ & 0.9 & $0.87(0.13-5.8)$ & 0.89 \\
\hline Moderate (THI score 38-56) & $4.4(0.89-21.3)$ & 0.068 & $2.5(0.42-14.3)$ & 0.31 \\
\hline Severe (THI score 58-100) & $28.9(5.9-141)$ & $<0.001$ & $10.1(1.7-60.8)$ & 0.011 \\
\hline \multicolumn{5}{|l|}{ Hyperacusis handicap } \\
\hline No $(H Q<22)$ & 1.0 & & 1.0 & \\
\hline Yes $(H Q \geq 22)$ & $4.1(2.2-7.7)$ & $<0.001$ & $2.2(0.87-5.4)$ & 0.097 \\
\hline \multicolumn{5}{|l|}{ Hyperacusis } \\
\hline No (ULLmin >77 dB HL) & 1.0 & & 1.0 & \\
\hline Yes (ULLmin $\leq 77$ dB HL) & $1.98(1.1-3.6)$ & 0.022 & $0.65(0.24-1.7)$ & 0.38 \\
\hline \multicolumn{5}{|l|}{ Depression } \\
\hline Normal $(\mathrm{PHQ}-9<10)$ & 1.0 & & 1.0 & \\
\hline Abnormal (PHQ-9 $\geq 10)$ & $21.2(9.98-44.99)$ & $<0.001$ & $6.8(2.8-16.3)$ & $<0.001$ \\
\hline
\end{tabular}


after adjustment for the effect of other variables, this association was not statistically significant $(\mathrm{OR}=1.1$, $p=0.83)$.

Table 3 (columns 2 and 3 ) shows that the risk of anxiety, indicated by a GAD-7 score $\geq 8$, increased 2.6-fold for patients with a childhood history of parental mental illness $(p=0.002)$. However, when the model was adjusted for the effects of other variables in the model plus age and gender (columns 4 and 5, Table 3 ), the relationship between parental mental health and anxiety was no longer significant (adjusted OR $=2.1,95 \%$ CI: $0.89-4.9, p=0.088$ ). In the adjusted model, the risk of anxiety was increased by a factor of 10.1 ( $p=$ 0.011 ) for those with severe tinnitus handicap (THI score 58-100) and by a factor of $6.8(p<0.001)$ for those with depression. Without adjustment for other variables, hyperacusis as diagnosed by $\mathrm{HQ} \geq 22$ and ULLmin $\leq 77 \mathrm{~dB}$ HL was significantly associated with anxiety (unadjusted $\mathrm{OR}=4.1, p<0.001$ for the $\mathrm{HQ}$ and $1.98, p=0.022$ for ULLmin). However, these associations were not statistically significant in the adjusted model (adjusted $\mathrm{OR}=2.2, p=0.097$ for the $\mathrm{HQ}$ and $0.65, p=0.38$ for ULLmin).

\section{DISCUSSION}

$\mathrm{T}$ he main aim of this study was to investigate, in a population of patients seeking help for their tinnitus and/or hyperacusis from a specialized audiology service in the United Kingdom, whether poor parental mental health in childhood was associated with anxiety and depression in adulthood. Thirty-nine percent of patients reported that during the first 18 years of their life, their parent(s) suffered from mental illness. This is higher than the prevalence of parental mental illness based on surveys of the general population, estimates of which range from $17.5 \%$ (Felitti et al, 1998) to about 19.5\% (Anda et al, 2006; Chapman et al, 2013). This discrepancy probably reflects differences in the study populations. Our sample consisted of patients seeking help for tinnitus and/or hyperacusis, with $42 \%$ having depression symptoms and $47 \%$ experiencing anxiety, which is much higher than the prevalence of depression and anxiety in general population. The prevalence of depression and anxiety as measured via the PHQ-9 and GAD-7 for the general population is about $7 \%$ and 6\%, respectively (Lowe et al, 2008; Hinz et al, 2016). Those with anxiety and depression are more likely to have been exposed to ACE (Anda et al, 2006). Our analyses suggest that the high incidence of depression and anxiety in our sample of patients may be partly linked to parental mental illness and partly linked to their tinnitus and hyperacusis. A clinical implication for audiologists who offer support and therapy for patients experiencing tinnitus and/or hyperacusis is that patients should be screened for pa- rental mental illness (via the question used in this study, which is adopted from the ACE questionnaire) and for problems such as anxiety and depression (via the GAD-7 and PHQ-9). There are at present no cures for tinnitus or hyperacusis. CBT has been shown to be effective in helping patients to reduce the impact of tinnitus and/or hyperacusis on their lives (Hesser et al, 2011; Aazh et al, 2016; Aazh and Moore, 2018a). However, patients with a history of ACE may need more complex and in-depth psychological treatments should they develop emotional problems (Pigeon et al, 2009; Kajeepeta et al, 2015).

It is important to bear in mind that parental mental illness in childhood may be associated with other childhood adversities. People are very often exposed to more than one category of ACE. For example, Felitti et al (1998) reported that for respondents reporting any single category of ACE, the probability of exposure to any additional category ranged from $65 \%$ to $93 \%$. In particular, among those who reported parental mental illness, $30 \%$ also reported psychological abuse, $22 \%$ reported physical abuse, 37\% reported sexual abuse, $46 \%$ reported substance abuse in the family, $26 \%$ reported their mother being treated violently, and $7 \%$ reported that a family member was imprisoned. Overall, of those who reported parental mental illness, $74 \%$ also reported at least one additional type of ACE exposure (Felitti et al, 1998). Felitti et al (1998) reported that the OR for depression (adjusted for age, gender, race, and education) was 1.5 (95\% CI: 1.3-1.7) for those with one adverse type of exposure in their childhood and 4.6 (95\% CI: 3.8-5.6) for those with four or more adverse types. It is very important to identify a history of ACE so that proper treatment and care can be provided by mental health professionals (Kajeepeta et al, 2015).

In our study, the mean PHQ-9 and GAD-7 scores were worse for patients with a history of parental mental illness than for those without, by 3.8 points and 2.8 points, respectively (both $p<0.05$ ). This is consistent with the findings of Chapman et al (2013) in a survey conducted on the general population, who reported that frequent mental distress was present for $8.8 \%$ of participants with no history of parental mental illness compared with $19.3 \%$ of those with such a history. Frequent mental distress was considered as present if respondents answered " 14 days or more" to the question "Now thinking about your mental health, which includes stress, depression, and problems with emotions, for how many days during the past 30 days was your mental health not good?" Our results are consistent with studies suggesting that poor parental mental health is a risk factor for a wide range of psychological disorders throughout the life span (Beesdo-Baum and Knappe, 2012; Beesdo-Baum et al, 2015). This could be partly related to the heritability of some of the mental health disorders. In addition, if a parent suffers from 
mental illness, this will have an effect on their relationship with their partner and their children, and this could lead to poorer mental health among the children (Anda et al, 2006).

In our study, parental mental illness in childhood was significantly associated with the risk of depression as measured via the PHQ-9, even after adjusting for the effect of age, gender, THI category, HQ score, ULLmin, and GAD-7 score. The adjusted OR was 2.7 (95\% CI: $1.08-6.7, p=0.033$ ). This indicates that patients with tinnitus and/or hyperacusis are at higher risk of developing depression if they have a childhood history of parental mental illness than if they do not. To the authors' knowledge, this finding has not previously been reported.

Our study was a retrospective analysis of clinical data. Hence, it was limited to information that was gathered in day-to-day clinical practice, and the only question about ACE was concerned with parental mental illness. Future research should systematically examine the relationship between the range of $\mathrm{ACE}$ and anxiety and depression among patients with tinnitus and hyperacusis.

\section{Study Limitations}

As this was a retrospective study, the analyses conducted here do not directly indicate that poor parental mental health in childhood leads to a high incidence of anxiety and depression in adulthood among people seeking help for tinnitus and hyperacusis. Causal relationships cannot be inferred from a study with our design.

In this study, $85 \%$ of the patients had been seen by an ENT specialist and $100 \%$ had been seen by their general practitioner before being referred to the THTSC. Our results may not be representative of patients who self-refer for treatment for tinnitus and hyperacusis. Nevertheless, our data are relevant to audiology services offering rehabilitative interventions for patients with troublesome tinnitus or hyperacusis who seek professional help in the management of their symptoms. Such patients form the typical caseload of audiologists who specialize in tinnitus and hyperacusis rehabilitation.

\section{CONCLUSIONS}

$\mathrm{T}$ hirty-nine percent of patients seeking help for their tinnitus and/or hyperacusis reported a history of parental mental illness in their childhood, which is a higher incidence than for the general population. This is important because parental mental illness in childhood may be associated with other childhood adversities. Therefore, audiologists who offer support and therapy for patients experiencing tinnitus and/or hyperacusis should screen for parental mental illness and refer a patient for further psychological assessment and treatment when needed. Our results showed that parental mental illness in childhood increased the risk of depression by a factor of 2.7 for patients with tinnitus and/or hyperacusis. Therefore, screening for parental mental illness in childhood is even more important among patients who suffer from symptoms of depression in addition to tinnitus and/or hyperacusis.

Future research should systematically examine the relationship between the different types of $\mathrm{ACE}$ and the anxiety and depression experienced by patients with tinnitus and/or hyperacusis. It should also explore whether the outcome of CBT for tinnitus and/or hyperacusis differs for patients with and without a history of childhood adversities.

Acknowledgments. We thank members of the Tinnitus and Hyperacusis Therapy Specialist Clinic at Guildford for their help in data collection. We thank two anonymous reviewers for their comments on earlier versions of the paper.

\section{REFERENCES}

Aazh H, Allott R. (2016) Cognitive behavioural therapy in management of hyperacusis: a narrative review and clinical implementation. Aud Vest Res 25:63-74.

Aazh H, Langguth B, Danesh AA. (2018) Parental separation and parental mental health in childhood and tinnitus and hyperacusis disability in adulthood: a retrospective exploratory analysis. Int $J$ Audiol 57(12):941-946.

Aazh H, McFerran D, Salvi R, Prasher D, Jastreboff M, Jastreboff M. (2014) Insights from the first international conference on hyperacusis: causes, evaluation, diagnosis and treatment. Noise Health 16:123-126.

Aazh H, Moore BCJ. (2016) A comparison between tinnitus retraining therapy and a simplified version in treatment of tinnitus in adults. Aud Vest Res 25:14-23.

Aazh H, Moore BCJ. (2017a) Factors associated with depression in patients with tinnitus and hyperacusis. Am J Audiol 26:562-569.

Aazh H, Moore BCJ. (2017b) Factors related to Uncomfortable Loudness Levels for patients seen in a tinnitus and hyperacusis clinic. Int J Audiol 56:793-800.

Aazh H, Moore BCJ. (2017c) Incidence of discomfort during puretone audiometry and measurement of uncomfortable loudness levels among people seeking help for tinnitus and/or hyperacusis. Am J Audiol 26:226-232.

Aazh H, Moore BCJ. (2017d) Usefulness of self-report questionnaires for psychological assessment of patients with tinnitus and hyperacusis and patients' views of the questionnaires. Int $J$ Audiol 56:489-498.

Aazh H, Moore BCJ. (2018a) Effectiveness of audiologist-delivered cognitive behavioral therapy for tinnitus and hyperacusis rehabilitation: outcomes for patients treated in routine practice. $A m J$ Audiol 27(4):547-558.

Aazh H, Moore BCJ. (2018b) Prevalence and characteristics of patients with severe hyperacusis among patients seen in a tinnitus and hyperacusis clinic. J Am Acad Audiol 29:626-633. 
Aazh H, Moore BCJ. (2018c) Proportion and characteristics of patients who were offered, enrolled in and completed audiologist-delivered cognitive behavioural therapy for tinnitus and hyperacusis rehabilitation in a specialist UK clinic. Int $J$ Audiol 57:415-425.

Aazh H, Moore BCJ. (2018d) Thoughts about suicide and selfharm in patients with tinnitus and hyperacusis. J Am Acad Audiol 29:255-261.

Aazh H, Moore BCJ, Glasberg BR. (2008) Simplified form of tinnitus retraining therapy in adults: a retrospective study. $B M C$ Ear Nose Throat Disord 8:1-7.

Aazh H, Moore BCJ, Lammaing K, Cropley M. (2016) Tinnitus and hyperacusis therapy in a UK national health service audiology department: patients' evaluations of the effectiveness of treatments. Int $J$ Audiol 55:514-522.

Alcala HE, Tomiyama AJ, von Ehrenstein OS. (2017) Gender differences in the association between adverse childhood experiences and cancer. Womens Health Issues 27:625-631.

Anda R, Tietjen G, Schulman E, Felitti V, Croft J. (2010) Adverse childhood experiences and frequent headaches in adults. Headache 50:1473-1481.

Anda RF, Brown DW, Dube SR, Bremner JD, Felitti VJ, Giles WH. (2008) Adverse childhood experiences and chronic obstructive pulmonary disease in adults. Am J Prev Med 34:396-403.

Anda RF, Brown DW, Felitti VJ, Bremner JD, Dube SR, Giles WH. (2007) Adverse childhood experiences and prescribed psychotropic medications in adults. Am J Prev Med 32:389-394.

Anda RF, Dong M, Brown DW, Felitti VJ, Giles WH, Perry GS, Valerie EJ, Dube SR. (2009) The relationship of adverse childhood experiences to a history of premature death of family members. BMC Public Health 9:106.

Anda RF, Felitti VJ, Bremner JD, Walker JD, Whitfield C, Perry BD, Dube SR, Giles WH. (2006) The enduring effects of abuse and related adverse experiences in childhood. A convergence of evidence from neurobiology and epidemiology. Eur Arch Psychiatr Clin Neurosci 256:174-186.

Andersson G, Carlbring P, Kaldo V, Strom L. (2004) Screening of psychiatric disorders via the Internet. A pilot study with tinnitus patients. Nord J Psychiatry 58:287-291.

Beesdo-Baum K, Knappe S. (2012) Developmental epidemiology of anxiety disorders. Child Adolesc Psychiatr Clin N Am 21: $457-478$.

Beesdo-Baum K, Knappe S, Asselmann E, Zimmermann P, Bruckl T, Höfler M, Behrendt S, Lieb R, Wittchen HU. (2015) The 'Early Developmental Stages of Psychopathology (EDSP) study': a 20-year review of methods and findings. Soc Psychiatry Psychiatr Epidemiol 50:851-866.

Bohman H, Laftman SB, Paaren A, Jonsson U. (2017) Parental separation in childhood as a risk factor for depression in adulthood: a community-based study of adolescents screened for depression and followed up after 15 years. BMC Psychiatry 17:117.

BSA. (2011a) Pure-Tone Air-Conduction and Bone-Conduction Threshold Audiometry with and without Masking: Recommended Procedure. Reading, United Kingdom: British Society of Audiology.

BSA. (2011b) Recommended Procedure: Determination of Uncomfortable Loudness Levels. Reading, United Kingdom: British Society of Audiology.
Chapman DP, Liu Y, Presley-Cantrell LR, Edwards VJ, Wheaton AG, Perry GS, Croft JB. (2013) Adverse childhood experiences and frequent insufficient sleep in 5 U.S. States, 2009: a retrospective cohort study. BMC Public Health 13:1-9.

Dong M, Giles WH, Felitti VJ, Dube SR, Williams JE, Chapman DP, Anda RF. (2004) Insights into causal pathways for ischemic heart disease: adverse childhood experiences study. Circulation 110: 1761-1766.

Dube SR, Anda RF, Felitti VJ, Chapman DP, Williamson DF, Giles WH. (2001) Childhood abuse, household dysfunction, and the risk of attempted suicide throughout the life span: findings from the adverse childhood experiences study. JAMA 286:3089-3096.

Dube SR, Williamson DF, Thompson T, Felitti VJ, Anda RF. (2004) Assessing the reliability of retrospective reports of adverse childhood experiences among adult HMO members attending a primary care clinic. Child Abuse Negl 28:729-737.

Felitti VJ, Anda RF, Nordenberg D, Williamson DF, Spitz AM, Edwards V, Koss MP, Marks JS. (1998) Relationship of childhood abuse and household dysfunction to many of the leading causes of death in adults. The adverse childhood experiences (ACE) study. Am J Prev Med 14:245-258.

Henry JA, Frederick M, Sell S, Griest S, Abrams H. (2015) Validation of a novel combination hearing aid and tinnitus therapy device. Ear Hear 36:42-52.

Hesser H, Weise C, Westin VZ, Andersson G. (2011) A systematic review and meta-analysis of randomized controlled trials of cognitive-behavioral therapy for tinnitus distress. Clin Psychol Rev 31: $545-553$.

Hinz A, Mehnert A, Kocalevent RD, Brahler E, Forkmann T, Singer S, Schulte T. (2016) Assessment of depression severity with the PHQ-9 in cancer patients and in the general population. BMC Psychiatry 16:22.

IAPT. (2011) IAPT Data Handbook v2.0.1 Appendices. London, United Kingdom: National IAPT Programme Team.

Jastreboff PJ, Jastreboff MM. (2003) Tinnitus retraining therapy for patients with tinnitus and decreased sound tolerance. Otolaryngol Clin North Am 36:321-336.

Jones TM, Nurius P, Song C, Fleming CM. (2018) Modeling life course pathways from adverse childhood experiences to adult mental health. Child Abuse Negl 80:32-40.

Juris L, Andersson G, Larsen HC, Ekselius L. (2013) Psychiatric comorbidity and personality traits in patients with hyperacusis. Int J Audiol 52:230-235.

Kajeepeta S, Gelaye B, Jackson CL, Williams MA. (2015) Adverse childhood experiences are associated with adult sleep disorders: a systematic review. Sleep Med 16:320-330.

Khalfa S, Dubal S, Veuillet E, Perez-Diaz F, Jouvent R, Collet L. (2002) Psychometric normalization of a hyperacusis questionnaire. ORL J Otorhinolaryngol Relat Spec 64:436-442.

Kroenke K, Spitzer RL, Williams JB. (2001) The PHQ-9: validity of a brief depression severity measure. J Gen Intern Med 16:606-613.

Langguth B, Landgrebe M, Kleinjung T, Sand GP, Hajak G. (2011) Tinnitus and depression. World J Biol Psychiatry 12:489-500.

Lee RD, Chen J. (2017) Adverse childhood experiences, mental health, and excessive alcohol use: examination of race/ethnicity and sex differences. Child Abuse Negl 69:40-48. 
Lowe B, Decker O, Muller S, Brahler E, Schellberg D, Herzog W, Herzberg PY. (2008) Validation and standardization of the generalized anxiety disorder screener (GAD-7) in the general population. Med Care 46:266-274.

Newman CW, Jacobson GP, Spitzer JB. (1996) Development of the tinnitus handicap inventory. Arch Otolaryngol Head Neck Surg 122:143-148.

Newman CW, Sandridge SA, Jacobson GP. (1998) Psychometric adequacy of the tinnitus handicap inventory (THI) for evaluating treatment outcome. J Am Acad Audiol 9:153-160.

Pattyn T, Van Den Eede F, Vanneste S, Cassiers L, Veltman DJ, Van De Heyning P, Sabbe BCG. (2016) Tinnitus and anxiety disorders: a review. Hear Res 333:255-265.

Paulin J, Andersson L, Nordin S. (2016) Characteristics of hyperacusis in the general population. Noise Health 18:178-184.

Pigeon WR, May PE, Perlis ML, Ward EA, Lu N, Talbot NL. (2009) The effect of interpersonal psychotherapy for depression on insom- nia symptoms in a cohort of women with sexual abuse histories. $J$ Trauma Stress 22:634-638.

Pinto PC, Marcelos CM, Mezzasalma MA, Osterne FJ, de Melo Tavares de Lima MA, Nardi AE. (2014) Tinnitus and its association with psychiatric disorders: systematic review. J Laryngol Otol 128:660-664.

Schecklmann M, Landgrebe M, Langguth B. (2014), TRI Database Study Group. (2014) Phenotypic characteristics of hyperacusis in tinnitus. PLoS One 9:e86944.

Spitzer RL, Kroenke K, Williams JB, Lowe B. (2006) A brief measure for assessing generalized anxiety disorder: the GAD-7. Arch Intern Med 166:1092-1097.

Tyler RS, Stouffer JL, Schum R. (1989) Audiological rehabilitation of the tinnitus client. J Acad Rehabil Audiol 22: 30-42.

Zigmond AS, Snaith RP. (1983) The hospital anxiety and depression scale. Acta Psychiatr Scand 67:361-370. 